\title{
A FREQUENCY REFERENCE FOR CARRIER SYNTHESIS IN WIRELESS STANDARD 802.11G
}

\author{
IULIAN URSAC, FLORIN CONSTANTINESCU, MIHAI MARIN \\ Department of Electrical Engineering, University Politehnica of Bucharest, Romania \\ E-mail: ursac.iulian@gmail.com
}

\begin{abstract}
A new approach to a frequency synthesizer is proposed: instead of a fixed frequency reference oscillator and a variable ratio frequency divider we use a variable frequency reference oscillator and a fixed ratio frequency divider. The implementation and characterization of a low phase-noise compensated oscillator used as frequency reference for a wireless transceiver is described. This circuit employs a high $Q$ piezoelectric resonator together with a CMOS cross coupled pair amplifier. A calibration circuit for compensation of frequency errors with respect to process variation is proposed.
\end{abstract}

Keywords: frequency synthesizer, voltage-controlled oscillator, phase locked loop, BAW AIN resonator

\section{INTRODUCTION}

\subsection{PLL synthesizer}

Many high frequency applications as local oscillators for various communication links or network analyzers require the synthesis of a signal having tight error margins for parameters as frequency, settling time and phase noise. A solution to this problem is the phase locked loop (PLL) synthesizer, whose block diagram is given in Figure 1 [1].

The desired signal is provided by the voltage-controlled oscillator (VCO) at its terminal Fout. Usually the frequency reference (FREF) is a quartz oscillator, known for its stability of the oscillation frequency.

The phase and frequency detector (PFD) compares the reference signal REF with the divided oscillator signal available at Fout of the frequency divider (DIV). PFD gives the UP command if Fref $>$ (Fout of DIV) or the DN (down) command if FREF $<$ (Fout of DIV .

The charge pump (CP) can give at its terminal OUT a positive or a negative current. After being filtered by a low pass filter (LPF), this signal is applied to the VCTRL terminal of the voltage-controlled oscillator (VCO). The positive current increases the oscillation frequency of VCO, while the negative current diminishes its value. PFD has a "dead zone" corresponding to no UP or DOWN output signal (see Figure 1).

Usually, FREF is an oscillator providing a constant oscillation frequency. In the classical solution the desired VCO frequencies are those imposed for the transmission channels of wireless standard 802.11g, which are obtained with different division ratios of DIV, while the FREF has a constant frequency.

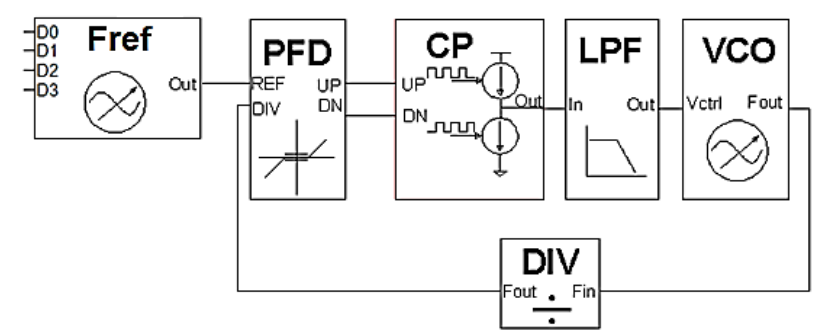

Figure 1. Block diagram of a PLL synthesizer

A new approach with respect of the above one is proposed in this paper, namely a PLL frequency synthesizer using a frequency reference which provides a signal having a set of frequencies, so that the desired set of VCO frequencies can be obtained with a divider whose division ratio remains unchanged.

The block diagram for this approach is that in Figure 1, too. The differences with respect to the classical solution are: the frequency reference gives an output with variable frequency, and the division factor of DIV is constant.

This solution has the advantage of a reduced phase noise with respect to the synthesizer with a variable division ratio [2]. Moreover, the classical quartz resonator is replaced by an AlN bulk acoustic wave (BAW) resonator which can be built in the same system on a chip (SoC) with the electronic MOS circuit, due to the high compatibility between these technologies [3].

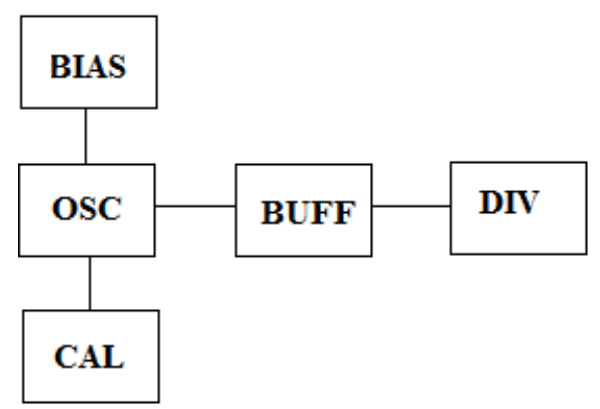

Figure 2. Block diagram of the frequency reference

In this case the frequency reference block Fref contains the blocks in Figure 2: BIAS (the circuitry for DC supply), OSC (reference oscillator), CAL (calibration circuit), BUFF (output buffer), and DIV (frequency divider).

The DC polarization (BIAS) of the active circuit is made using a Widlar cascoded voltage reference, a $3.3 \mathrm{~V}$ voltage supply and has a total current consumption of $2.64 \mathrm{~mA}$. 
The buffer circuit (BUFF) it is a PMOS differential pair amplifier with a NMOS current mirror active load and a PMOS current source, having a $5 \mathrm{~V}$ supply voltage and $4.04 \mathrm{~mA}$ current consumption.

The divider by 10 (DIV) is made with two classical dividers, a divider by 2 followed by a divider by 5 .

\subsection{BAW AIN resonator}

Any oscillator contains a sub-circuit with a selective frequency characteristic which controls the oscillation frequency. In this case this is the AlN power BAW resonator.

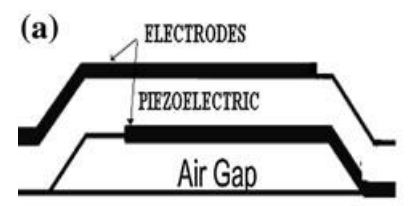

SUBSTRATE (b)

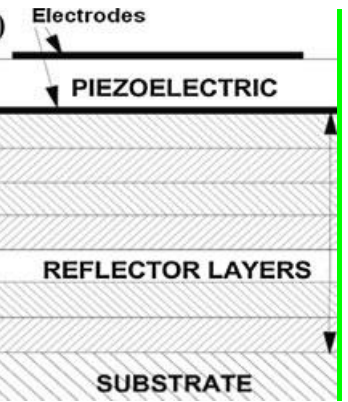

Figure 3. BAW resonator a) FBAR, b) SMR

A BAW resonator can be built as a thin-film bulk acoustic resonator (FBAR) structure or as a solidly mounted resonator (SMR) structure, as it is shown in Figure 3. In order to not use the mechanical wave energy for heating the substrate, FBAR uses an air gap while SMR employs a Bragg mirror built using alternative layers of low and high mechanical impedances [3].

In the small signal operation, a structure of this kind has the linear behavior shown in Figure 4.a and the equivalent circuit given in Figure 4. B [3,4].

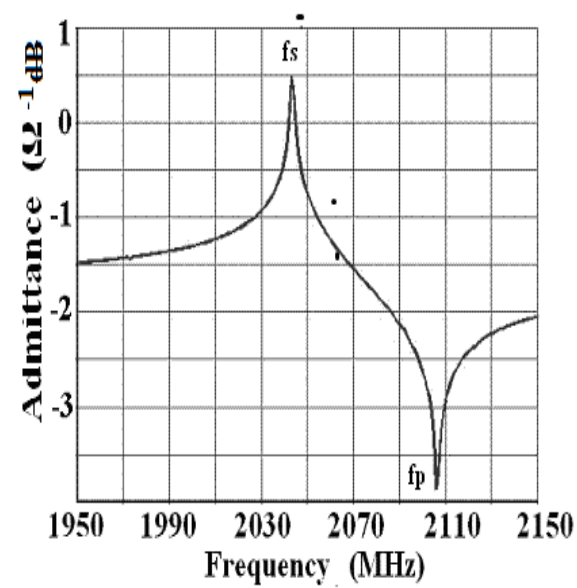

a

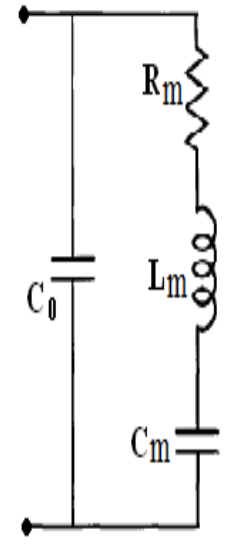

b
Figure 4. Small signal behavior of an AIN BAW resonator a) frequency characteristic, b) linear equivalent circuit

If a certain input power level is exceeded the nonlinear equivalent circuit in Figure 5 can be used $[5,6]$.

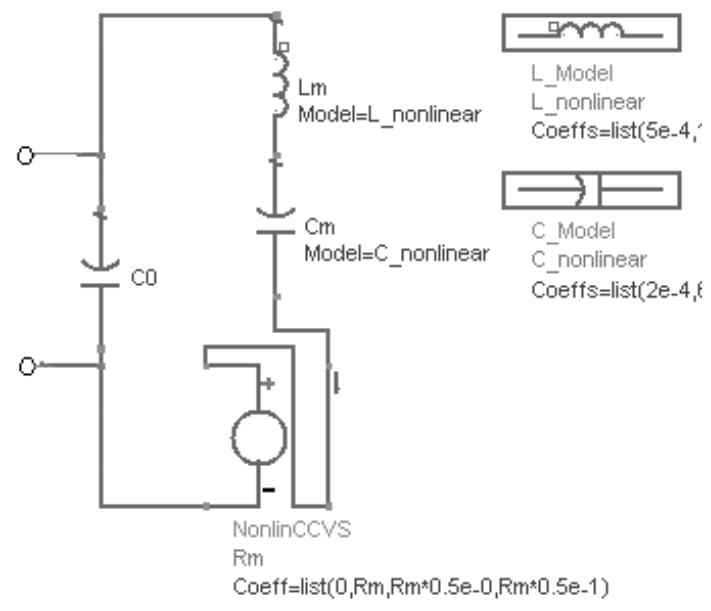

Figure 5. Nonlinear equivalent circuit for a power BAW resonator

The design of the blocks of the frequency reference are presented in Section 2, and its performances are described in Section 3.

\section{FREQUENCY REFERENCE DESIGN}

\subsection{Active circuit}

We use the cross coupled differential-pair (M1 and M2 in Figure 6) oscillator in order to provide the negative conductance which allows the oscillations to exist.

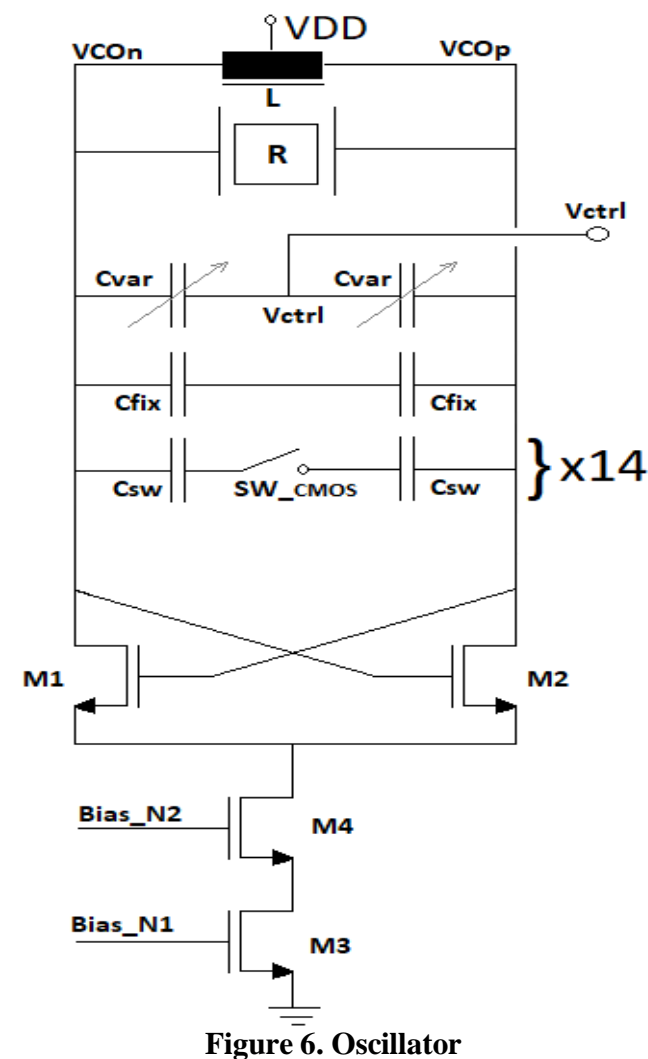

As our intention is to generate 14 carrier frequencies required by the wireless standard $802.11 \mathrm{~g}$, a procedure for changing the oscillation frequency is needed. For the cross coupled differential-pair schematic the oscillation 
frequency will be the parallel resonance frequency $f_{p}$ of the circuit containing the BAW resonator $\mathrm{R}$ together with $\mathrm{L}$ and the capacitors in Figure 6. The circuit a.c. impedance has the maximum module value at $\mathrm{f}_{\mathrm{p}}$ allowing M1 and M2 to alternate their conduction states.

Two schematics are presented in [7, 8] aimed to modify the parallel resonance frequency $\mathrm{f}_{\mathrm{p}}$ of a BAW resonator. (a)

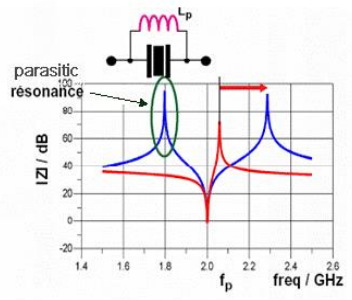

(b)

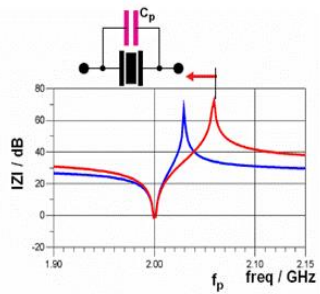

Figure 7. Schematics for $f_{p}$ modification

Our choice is the use of a parallel coil (Figure 7 a) which allows the bias current to reach the transistors M1 and M2 in Figure 6. In this case a parasitic parallel resonance frequency is generated (Figure 7a), and the oscillation frequency will be that corresponding to the maximum $|z|$ value, corresponding to a minimum loss in this circuit.

It is obvious from Figure $7 \mathrm{~b}$ that connecting a supplementary capacitor in parallel with the resonator, the value of $\mathrm{fp}$ corresponding to the whole circuit is diminished. In order to generate all oscillation frequencies for the wireless standard $802.11 \mathrm{~g}$, our design will start from the maximum frequency, connecting parallel trimming capacitors as in Figure 6, in order to obtain lower and lower oscillation frequencies (see Table 1).

For a linear BAW resonator there are some formulas giving $f_{s}$ and $f_{p}$ as functions of the parameters in the linear model in Figure 4b [4]. For the structures in Figs. 7a and $7 \mathrm{~b}$, this kind of formulas don't exist. This is because we use the transient analysis of CADENCE to verify the value of the highest parallel resonance frequency for the circuit in Figure 6.

The capacitors $\mathrm{C}_{\mathrm{var}}, \mathrm{C}_{\text {fix }}$, and the $14 \mathrm{C}_{\mathrm{sw}}$ pairs in Figure 6 are chosen from the library of the design kit for the TSMC $180 \mathrm{~nm}$ technology. In order to reduce the influence of the parasitic capacities of this circuit (CMOS layout connections with respect to ground or vias through BAW/CMOS separation layer) two fixed value capacities are considered. Their capacitance value is $\mathrm{C}_{\text {fix }}=394 \mathrm{fF}$ [9]. Aiming to compensate the process variations from one sample circuit to another, a $\mathrm{C}_{\mathrm{var}}$ capacity pair which is controlled by the $\mathrm{V}_{\text {ctrl }}$ voltage is added (Figure 6). In our TSMC 180nm technology the maximum range of a variable capacity (varactor diode) is ( $442 \mathrm{fF}-547 \mathrm{fF})$.

As, having no available BAW AlN technology, we cannot design this resonator, we start from a device fabricated by CEA-LETI [6], whose parameters will be modified in order to be suited to our purpose. The resonator A in [6] has the series and parallel resonance frequencies $\mathrm{f}_{\mathrm{s}}=2.033$ $\mathrm{GHz} \mathrm{fp}=2.081 \mathrm{GHz}$, the low frequency capacity $\mathrm{Clf}=1.824$ $\mathrm{pF}$ and the quality factor at $\mathrm{f}_{\mathrm{s}} \mathrm{Qs}=1000$. It has also the AlN thickness $b=1.17 \mu \mathrm{m}$. In order to change the parallel frequency value to $\mathrm{f}_{\mathrm{p} 1}=0.95 \mathrm{GHz}, \mathrm{b}$ is changed to $\mathrm{b}_{1}=\mathrm{b} \cdot \mathrm{f}_{\mathrm{p}} / \mathrm{f}_{\mathrm{pl}}=2.5038 \mu \mathrm{m}$ [6]. The other parameters of the new resonator are: $\mathrm{f}_{\mathrm{s} 1}=\mathrm{f}_{\mathrm{p}} \operatorname{sqrt}\left(1-\mathrm{k}_{\mathrm{t}}^{2}\right)=0.928 \mathrm{GHz}$, $\mathrm{C}_{\mathrm{lf} 1}=\mathrm{C}_{\mathrm{lf}} \mathrm{f}_{\mathrm{p} 1} / \mathrm{f}_{\mathrm{p}}=0.8326 \mathrm{pF}, \mathrm{k}_{\mathrm{t}}^{2}=4.5 \%$; the parameters of the resonator equivalent circuit in Fig $4 \mathrm{~b}$ and Fig 5 can be computed using the values $\mathrm{Cm} 1=\mathrm{C}_{\mathrm{lf1}} * \mathrm{k}_{\mathrm{t}}^{2}=0.8326 *$ $0.0455=0.037 \mathrm{pF}, \mathrm{C}_{01}=\mathrm{C}_{\mathrm{lf} 1} *\left(1-\mathrm{k}_{\mathrm{t}}^{2}\right)=0.7947 \mathrm{pF}, \mathrm{L}_{\mathrm{m} 1}$ $=1 /\left(\mathrm{C}_{\mathrm{m} 1} * \omega_{\mathrm{s}}^{2}\right)=0.795 \mu \mathrm{H}$.

Using two metal layers we can build an inductor in the TSMC $180 \mathrm{~nm}$ technology (Figure 8). This structure is symmetric, the quality factor being less than 10 .

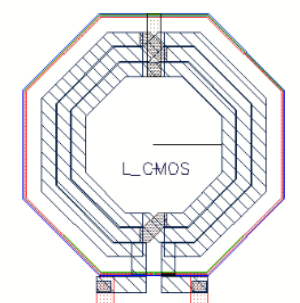

Figure 8. CMOS inductor layout

Consider all $\mathrm{C}_{\mathrm{sw}}$ disconnected and $\mathrm{C}_{\mathrm{var}}$ with the median value. In order to choose the best value of $\mathrm{L}$, some $\mathrm{AC}$ simulations of the circuit in Figure 6 have been performed with CADENCE, the results being shown in Figure 9. This circuit contains the equivalent circuit of the resonator (Figure 4. b) and some parasitic capacities $C_{\text {layout }}=100 \mathrm{fF}$, $\mathrm{C}_{\text {via }}=400 \mathrm{fF}$ [9], $\mathrm{C}_{\text {load }}=100 \mathrm{fF}, \mathrm{C}_{\text {tranz }}=\mathrm{C}_{\mathrm{gs}}=400 \mathrm{fF}$ whose meanings are obvious. The results of these simulations are given in Figure 9, the value of L being attached to each frequency characteristic.

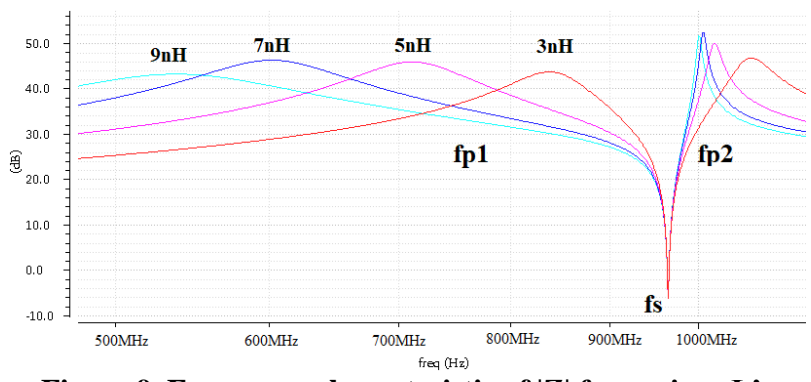

Figure 9. Frequency characteristic of $|\mathrm{Z}|$ for various $L$ 's

It follows that the optimal value is $\mathrm{L}=5 \mathrm{nH}$ because it provides a relatively good quality factor and a relatively high parallel resonance frequency in order to have enough tuning room. From the library of TSMC $180 \mathrm{~nm}$ design kit, it results that this coil has three turns, a quality factor $\mathrm{Q}=8.6$, and the area of $0.367 \mathrm{~mm}^{2}$.

As adding successively some capacitors $C_{\text {sw }}$ in parallel with the BAW resonator it is expected that $f_{p}$ for the circuit in Figure 6 will decrease, our design will start with the finding the $\mathrm{C}_{\mathrm{sw}}$ value for the maximum FREF frequency, namely 993.6 MHz (see Table 1). In principle, we could employ a capacitor for each channel, but the commutation of capacitors with relatively large values leads to a longer transient regime for reaching the new frequency steady state than the commutation of capacitors with smaller values which remain connected as we change the frequency from $f_{\max }$ to $f_{\min }$ by sweeping all channels. 
The identification of each of these capacity values is made using a sequence of attempts with various $C_{\mathrm{sw}}$ values, each attempt consisting in the transient analysis of the oscillator. After 3-5 attempts the value of the $\mathrm{C}_{\mathrm{sw}}$ ensuring the desired oscillation frequency is found. The next step is to determine the $\mathrm{C}_{\mathrm{sw}}$ value for the minimum frequency, which is the sum of all $\mathrm{C}_{\mathrm{sw}}$ values. Finally all $\mathrm{C}_{\mathrm{sw}}$ values are given in Table 1.

Table 1. Frequencies and trimming capacitors values

\begin{tabular}{|r|c|c|c|c|}
\hline \multicolumn{1}{|l|}{ CH } & $\begin{array}{c}\text { VCO Freq.. } \\
\text { Ref. [Mhz] }\end{array}$ & $\begin{array}{l}\text { Csw } \\
{[\mathrm{fF}]}\end{array}$ & $\begin{array}{c}\text { Divided } \\
\text { Freq. } \\
\text { Ref. } \\
{[\mathrm{Mhz}]}\end{array}$ & $\begin{array}{l}\text { PLL } \\
\text { Freq. } \\
{[\mathrm{MHz}]}\end{array}$ \\
\hline 14 & 964.8 & 167 & 96.48 & 2412 \\
\hline 13 & 966.8 & 394 & 96.68 & 2417 \\
\hline 12 & 968.8 & 231 & 96.88 & 2422 \\
\hline 11 & 970.8 & 234 & 97.08 & 2427 \\
\hline 10 & 972.8 & 236 & 97.28 & 2432 \\
\hline 9 & 974.8 & 238 & 97.48 & 2437 \\
\hline 8 & 976.8 & 240 & 97.68 & 2442 \\
\hline 7 & 978.8 & 242 & 97.88 & 2447 \\
\hline 6 & 980.8 & 244 & 98.08 & 2452 \\
\hline 5 & 982.8 & 246 & 98.28 & 2457 \\
\hline 4 & 984.8 & 249 & 98.48 & 2462 \\
\hline 3 & 986.8 & 251 & 98.68 & 2467 \\
\hline 2 & 988.8 & 253 & 98.88 & 2472 \\
\hline 1 & 993.6 & 269 & 99.36 & 2484 \\
\hline
\end{tabular}

\subsection{Calibration circuit}

The AlN BAW resonance frequencies are influenced by temperature and changes of the technological process. Two types of methods are applied for the frequency drift compensation with temperature of a BAW AlN oscillator working in a certain frequency band. The first one uses a passive compensation which can lead to a minimum relative error of $\pm 40 \mathrm{ppm}$ frequency shift over the entire frequency range [10]. The second one employs an active way of compensation and can lead to a minimum error of \pm 10 ppm over the entire frequency range [11].

The CMOS process changes can lead to variations up to $30 \%$ of capacity or resistance values. In the AlN BAW technology the piezoelectric layer thickness can vary from sample to sample leading to deviations of $\mathrm{f}_{\mathrm{s}}$ and $\mathrm{f}_{\mathrm{p}}$, also.

Some methods based on trimming using passive circuit elements which compensate OSC frequency errors are described in [12] and [13]. These methods are suitable just for one output frequency; for multiple frequencies their associate testing cost being increased by the trimming process.

Taking into account that a wireless transceiver is placed usually inside a building, this location having no significant temperature variations, a new approach to compensate frequency errors with respect to process variation is proposed. The block schematic of the oscillator calibration circuit is given in Figure 10.

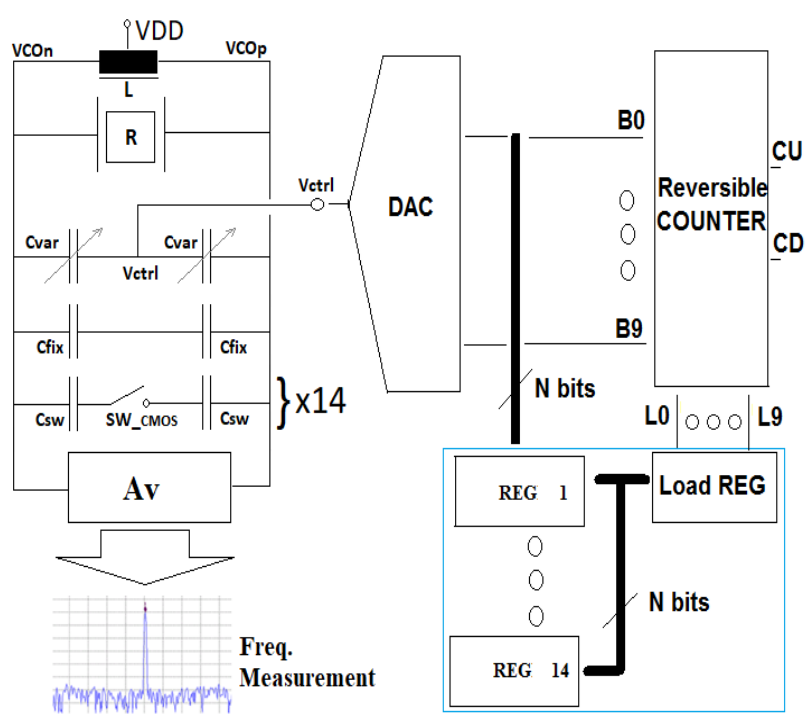

Figure 10. Block schematic of the calibration circuit

The upper left part of Figure 10 shows the capacitors in Figure 6, which are used to obtain the desired oscillation frequencies. The frequency reference calibration is performed for each of the 14 output communication channels when the wireless transceiver is tested. The channel is selected using the appropriate $\mathrm{C}_{\mathrm{sw}}$ fixed capacitor pair by closing the appropriate set of SW_CMOS switches. At each frequency we drive the Reversible COUNTER to count up (CU) or to count down (CD) in order to sweep the full range of $\mathrm{V}_{\text {ctrl }}$ values from the output of the digital to analog converter (DAC). The DAC output voltage controls the variable capacitors $C_{\text {var }}$ till the desired frequency is obtained. When the Spectrum Analyzer measures this value in the reference output, the reversible counter digital output is recorded in the appropriate nonvolatile register (REG1, ..., REG14). After the calibration process, the result is a 10 bits digital code for each channel frequency generated by this circuit.

In the normal operation of the frequency reference, when a communication channel is selected with a 4 bits digital word, the same word is used to select its 10 bits code for frequency calibration recorded in the nonvolatile memory. This code is loaded into a 10 bits load register (Load Reg) which controls the reversible counter, in order to command the DAC output voltage to drive variable capacitors $C_{\text {var }}$ to the values corresponding to the prescribed channel frequency.

\section{FREQUENCY REFERENCE PERFORMANCES}

\subsection{Spectral analysis}

Spectral analysis results of the reference frequency circuit for the unbuffered OSC, containing all 14 output frequencies is given in Figure 11. This picture gives a measure of spectral purity of the unbuffered OSC. 


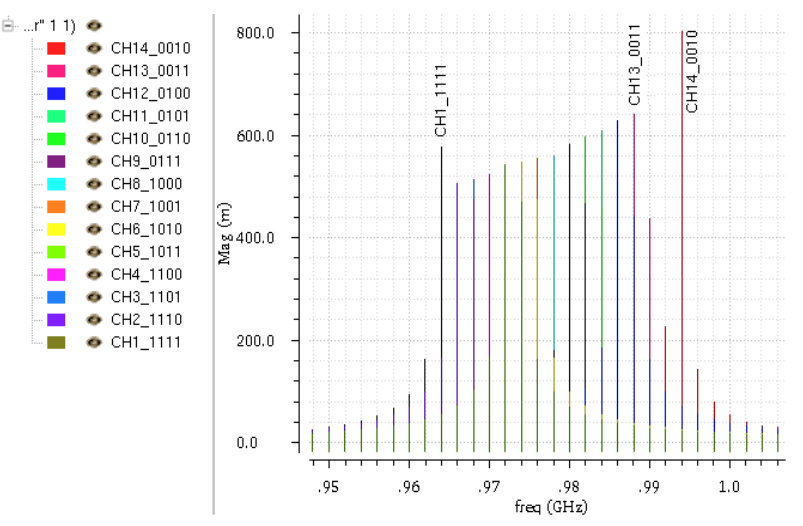

Figure 11. Spectral analysis of unbuffered OSC

We observe the ascendant trend of the oscillation amplitude for the last 13 frequencies. This effect can be explained by the lowering of the total Q factor determined by adding a new CMOS capacitor in parallel to a BAW resonator, due to the low $\mathrm{Q}$ of CMOS capacitors and to the capacitor switch resistance.

As BUF is an amplifier whose output signal is limited by its DC supply, its output signals have the same amplitude for all frequencies.

\subsection{Phase noise}

The $802.11 \mathrm{~g}$ standard specification regarding the phase noise requirement is described by Interference $=+35 \mathrm{~dB}$ (measured at $25 \mathrm{MHz}$ offset from the carrier). The phase noise analysis for all 14 channel frequencies, performed at the OSC output (Figure 2) is presented in Figure 12. These results are computed with PN analysis of CADENCE. The maximum value of the phase noise is $-176.7 \mathrm{dBc} / \mathrm{Hz}$ obtained for the channel 5 (Figure 12). This value complies with the specifications of the 802.11g standard [14].

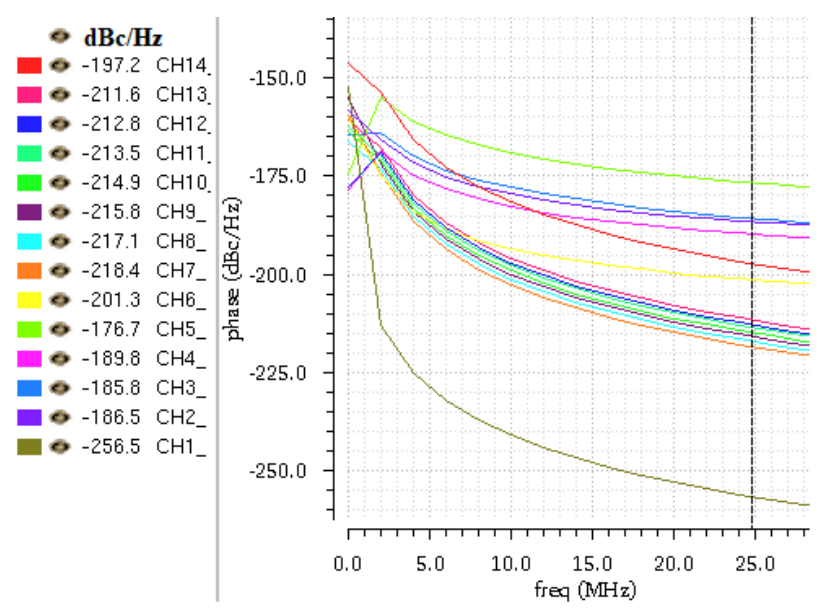

Figure12. Unbuffered OSC phase noise analysis

\subsection{Frequency stability}

In order to improve the frequency reference stability, the VCO is designed at high frequency, around $970 \mathrm{MHz}$. Using the frequency division by 10 the frequency deviation against median value is decreased 10 times. Starting from $802.11 \mathrm{~g}$ standard specification with \pm 25 ppm an allowed frequency deviation of $\pm 600 \mathrm{KHz}$ results in the range of $2.4 \mathrm{GHz}$. At the OSC output, where the simulation result in Figure 13 is given, this allowed frequency deviation is $\pm 24 \mathrm{KHz}$ (between $24.84 \mathrm{KHz}$ for $\mathrm{CH} 1$ and $24 \mathrm{KHz}$ for $\mathrm{CH} 14)$. The simulation results computed with the freq_jitter analysis of CADENCE are given in Figure 13 and comply with these limits.

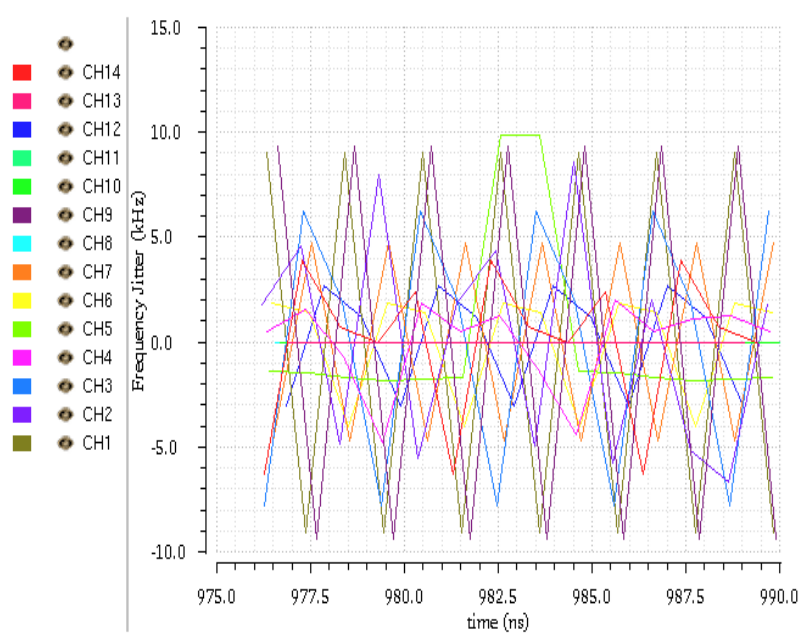

Figure13. Unbuffered VCO Jitter analysis

\subsection{Settling time}

The settling time according $802.11 \mathrm{~g}$ standard specification need to be $224 \mu$ s for the whole $2.4 \mathrm{GHz}$ frequency synthesizer. For the unbuffered frequency reference settling time we impose a limit below $1 \%$ $(2.24 \mu \mathrm{s})$ of the total settling time of the wireless transceiver. The maximum settling time is the time to change between communication channel frequencies; The start-up time represents the worst case. Next picture presents the simulation results for the worst case (start-up settling time) for all 14 VCO unbuffered output frequencies.

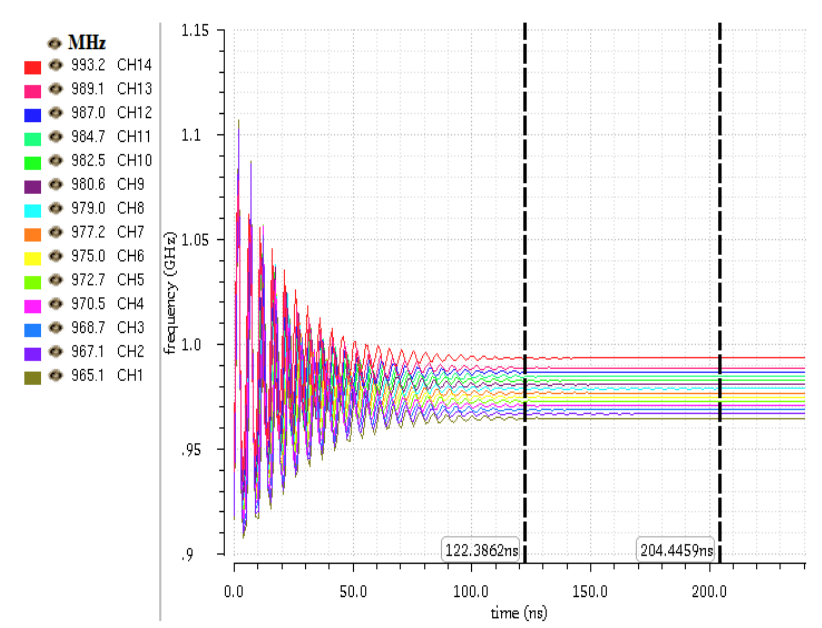

Figure14. Unbuffered OSC settling time analysis

We observe that settling time is different across output frequencies. The values are between $122.3 \mathrm{~ns}$ and $205 \mathrm{~ns}$ worst case, representing a value smaller than $10^{-3}$ from $224 \mu$ s (the total settling time of a frequency synthesizer given in the $802.11 \mathrm{~g}$ standard specification) [14]. 


\section{CONCLUSIONS}

The approach proposed in this paper has several advantages:

- The possibility to include the MOS circuitry together with the AlN BAW resonator in a system in a package (SiP).

- Using a frequency reference providing a variable frequency signal, the FDIV has a fixed division ratio for all channels of the wireless transceiver. This approach eliminates a main source of noise in the classical solution - the FDIV with variable division factor.

- A compensation circuit for the process variation has been proposed, in order to satisfy the specifications of the wireless standard $802.11 \mathrm{~g}$ regarding the parameters of the carrier signals for all 14 communication channels.

In order to prove these advantages, some simulations using several CADENCE analyses have been performed, using the design kit of the TSMC 180nm technology.

\section{REFERENCES}

[1] Collins I.,Phase-Locked Loop (PLL)Fundamentals, Analog Devices, 2018.

https://www.analog.com/en/landingpages/001/glp2-pll-funda-mentals-mt-086.html

[2] Baschirotto A.; Makinwa K. A.; Harpe P. Frequency references, power management for SoC, and smart wireless interfaces, Advances in analog circuit design 2013, Springer, 2014.

[3] Lakin K. M., Thin film resonator technologies. IEEE Trans UFFC, 52(5) 2005, pp.707-716.

[4] Nitescu M., Constantinescu F., Gheorghe A.-G., An A.C. linear parametric behavioral model of a nonlinear BAW resonator, International Conference on Circuits and Systems for Communications (ICCSC'06), July 10-11, 2006, Politehnica University, Bucharest, Romania.

[5] Constantinescu F., Nitescu M., Gheorghe A. G, Florea A., Llopis O., Behavioral circuit models of power BAW resonators and filters, Analog Integrated Circuits and Signal Processing, Springer, vol. 73 (2012), No. 1, pp. 57-64.

[6] Constantinescu F., Gheorghe A. G., Nitescu M., Florea A., Llopis O., Taras P., Parameter Identification for Nonlinear Circuit Models of Power BAW Resonators, Advances in Electrical and Computer Engineering Vol. 11, No. 1, 2011.

[7] Razafimandimby S., Accord en Fréquence de Résonateurs BAW Appliqué au Filtrage et à la Synthèse de Fréquence RF, Thèse de docteur, l'Université des Sciences et Technologies de Lille, 2007.

[8] Cathelin A., Razafimandimby S., Kaiser A., BAWIC Co-Integration Tunable Filters at $\mathrm{GHz}$ Frequencies, IEMN - ISEN, Lille, France, 2007.

[9] Zuo C., Sinha N., der Spiegel J. V., Piazza G., Multifrequency Pierce oscillators based on piezoelectric AlN contour-mode MEMS resonators, Proceedings of the 2008 International Frequency Symposium, pp. $402-407$.

[10] R. Tabrizian, M. Pardo, and F. Ayazi, A 27 MHZ Temperature compensated MEMS oscillator with sub-ppm instability, IEEE Int. Conf. on MEMS, 2012, pp. 23-26.

[11] Discera Corporation, "DSC1121 Datasheet." http://www.audentiagestion.fr/Microchip/DSC1101_DSC1121_Datashe et.pdf

[12] Kinget P., Integrated GHz Voltage - Controlled Oscillators, Bell Labs - Lucent Technologies Murray Hill.

[13] Cottier D., Oscillator Compensation Guide, Texas Instruments Application Report SPRAB84A-June 2010.

[14] Moon, S., T., Valero-Lopez, A. Y., SanchezSinencio, E., Fully Integrated Frequency Synthesizers: A Tutorial, International Journal of High-Speed Electronics and Systems, November 2011, pp. 1-23. 